\title{
A new flow focusing technique to produce very thin jets
}

\author{
A J Acero ${ }^{1}$, N Rebollo-Muñoz ${ }^{1}$, J M Montanero ${ }^{1}$, A M Gañán-Calvo ${ }^{2}$ \\ and $\mathbf{E} \mathbf{J}$ Vega $^{2}$ \\ ${ }^{1}$ Departamento de Ingeniería Mecánica, Energética y de los Materiales, Universidad de Extremadura, \\ E-06006 Badajoz, Spain \\ ${ }^{2}$ Departamento de Mecánica de Fluidos e Ingeniería Aeroespacial, Universidad de Sevilla, \\ E-41092 Sevilla, Spain \\ E-mail: evega@us.es
}

Received 18 October 2012, in final form 19 March 2013

Published 26 April 2013

Online at stacks.iop.org/JMM/23/065009

\begin{abstract}
A new technique is proposed in this paper to produce jets, droplets, and emulsions with sizes ranging from tens of microns down to the submicrometer scale. Liquid is injected at a constant flow rate through a hypodermic needle to form a film over the needle's outer surface. This film flows toward the needle tip until a liquid ligament is steadily ejected. Both the film motion and the liquid ejection are driven by the viscous and pressure forces exerted by a coflowing fluid stream. If this stream is a high-speed gas current, the outcome is a capillary jet which breaks up into droplets due to the Rayleigh instability. Micrometer emulsions are also produced by this instability mechanism when the injected liquid is focused by a viscous liquid stream. The minimum flow rates reached with the proposed technique are two orders of magnitude lower than those of the standard flow focusing configuration. This sharp reduction of the minimum flow rate allows one to form steady jets with radii down to the submicrometer scale. The stability of this new configuration is analyzed experimentally for both gas-liquid and liquid-liquid systems. In most of the cases, the loss of stability must be attributed to the liquid source because the critical Weber (capillary) number for the gas-liquid (liquid-liquid) case was significantly greater than the value corresponding to the convective/absolute instability transition in the jet.
\end{abstract}

(Some figures may appear in colour only in the online journal)

\section{Introduction}

The controlled production of very small fluid entities such as drops, bubbles, emulsions, and capsules has been extensively investigated over the last two decades because of its enormous relevance for a great variety of applications. Micrometer fluid particles of varied morphology have been produced by making use of surfactants [1], electrical forces [2], or thermal gradients [3]. Extensional coflows constitute a remarkable class of techniques in which pressure [4] and/or viscous [5-8] forces stretch an interface until a small jet is emitted.

In flow focusing [4], use is made of an outer coflowing stream to accelerate and steadily eject a liquid injected through a feeding capillary. Both the liquid jet and the outer stream cross an orifice located in front of the capillary and whose diameter is much larger than that of the microjet. If the outer stream is a high-speed gas current [4], the outcome is a capillary jet which breaks up into droplets due to the Rayleigh instability [9]. When the focusing effect is caused by a viscous liquid current, emulsions consisting of micrometer droplets are produced [10]. In this case, both the inner and outer liquids coflow downstream with almost the same speed until the interface pinches due to the growth of capillary waves too. Drops significantly larger than those predicted by the inviscid Rayleigh's theory [9] can be obtained due to the disparity between the two viscosities involved [11].

Steady jetting is obtained with flow focusing for a wide range of operational conditions. This technique is very attractive because it uses purely hydrodynamic means to produce monodisperse collections of micrometer drops at a 


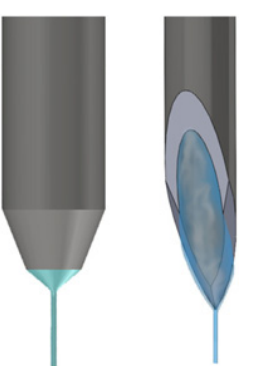

Figure 1. Standard capillary (left) and needle (right). The blue regions represent the liquid flow.

continuous high rate $[4,10]$. Stable high-speed drawing of fibers can also be obtained by focusing highly viscous jets with this coflowing arrangement [12]. The jet size is mainly controlled by selecting appropriately the liquid flow rate. In gaseous flow focusing, the pressure drop $\Delta p$ applied to the coflowing gas stream plays a secondary role in controlling the jet size. For both low and moderate-viscosity liquids, the jet diameter scales as $\Delta p^{-1 / 4}$. This means that reducing by a factor, say, 4 the diameter entails a $\Delta p$ increase of two orders of magnitude. In addition, it is well known that whipping instability arises when the difference between the speeds of outer and inner streams overcomes a certain threshold [13]. This aerodynamic instability breaks the jet in such a way that undesirable polydisperse collections of drops are produced.

The control of the jet diameter in flow focusing relies on the delicate balance between surface tension, viscous, and pressure forces that occurs in the tapering meniscus as the flow rate is varied. However, when this balance breaks down, the fluid configuration is no longer stable, and steady jetting can not be obtained. This occurs for sufficiently small liquid flow rates, which significantly limits the minimum size of the droplets produced. The mechanisms responsible for the meniscus instability are different in the low- and high-viscosity limits. While low-viscosity menisci become unstable because of the appearance of a recirculation cell [14], the loss of stability in the high-viscosity case is due to the resistance presented by the surface tension force [15]. Recently, a modified flow focusing configuration has been proposed to produce low viscosity microjets at significantly smaller flow rates than those reached by the standard configuration [16]. In the modified flow focusing device, a sharpened rod blocks the recirculation cell appearing in the tapering liquid meniscus for low flow rates, which considerably improves its stability.

In this paper, we propose a new technique for producing jets, droplets, and emulsions with sizes ranging from tens of microns down to the submicrometer scale. This technique is based on the flow focusing principle [4]. The main difference between the proposed and standard configurations is the device used to inject the focused liquid into the coflowing stream. The cylindrical capillary used in the classical flow focusing configuration [4, 10, 14, 15] is replaced with a (common) hypodermic needle of a similar dimension (see figure 1). The needle ends in a sharpened tip over which the injected liquid flows dragged by the outer current. In this way, the tapering meniscus of the standard flow focusing configuration is substituted by a Couette-type flow directed toward the needle
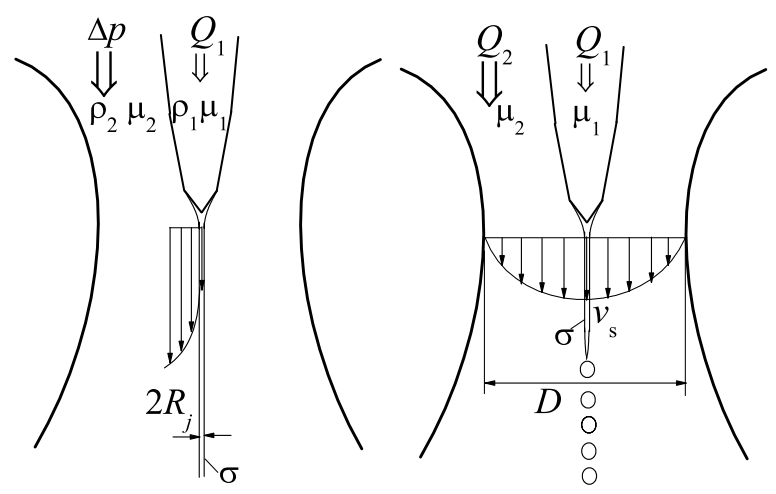

Figure 2. Sketch of gas-focused (left) and liquid-focused (right) jets, and the quantities introduced to describe the problem in each case.

tip. A liquid ligament is ejected from the very tip of the needle, which is only a few microns in size. This constitutes a fundamental change in the flow focusing method because it alters completely the dynamics and, therefore, the stability of the liquid source. Specifically, the absence of recirculation patterns for low and moderate-viscosity liquids enhances the flow stability, and prevents the liquid from dripping as the injected flow rate decreases. Hypodermic needles have been used previously to produce microjets in electrospray $[17,18]$. To the best our knowledge, the flow focusing principle is applied in combination with hypodermic needles here for the first time in the literature.

Our results will show that the new configuration is much more stable than the classical one as the liquid flow rate decreases. The minimum flow rates reached by the proposed technique are two orders of magnitude lower than those of the standard flow focusing for both gas-liquid [15] and liquid-liquid systems [10, 19]. In fact, the minimum flow rates reached with the new configuration have hardly ever been observed in techniques relying on purely hydrodynamic forces to produce microjets [19]. This drastic reduction of the minimum flow rate allows one to form extremely thin steady jets, whose radii can even fall below the $1 \mu \mathrm{m}$ barrier. It must be pointed out that such small jets are produced with orifices of about $200 \mu \mathrm{m}$ in diameter, thereby avoiding the clogging of the injection system.

The paper is organized as follows. The problem analyzed in this work is formulated in section 2 . This includes describing the considered fluid configurations (section 2.1), deriving the corresponding dimensionless numbers (section 2.2), and explaining our approach to the stability analysis of the problem (section 2.3). The experimental procedure is described in section 3 . In section 4 , we present the results for both the gasliquid (section 4.1) and liquid-liquid (section 4.2) systems. The paper closes with the conclusions in section 5 .

\section{Formulation of the problem}

\subsection{The fluid configurations}

The fluid configurations analyzed in this work are sketched in figure 2. A hypodermic needle is located in a convergingdiverging nozzle at a section of diameter $D$ much larger than 
the hydraulic radius $r_{t}$ of the needle tip. A liquid of density $\rho_{1}$ and viscosity $\mu_{1}$ is injected through the needle at a constant flow rate $Q_{1}$. The liquid slips over the needle's outer surface dragged by an outer fluid stream of density $\rho_{2}$ and viscosity $\mu_{2}$. The outer stream can be either a high-speed gas current driven by a pressure drop $\Delta p$, or a liquid viscous stream flowing at a constant rate $Q_{2}$. The former (latter) case corresponds to inertia (viscosity) dominated flows. In gaseous flow focusing, a thin boundary layer forms attached to the jet's free surface, while in the liquid-liquid configuration a quasi-parabolic velocity profile with a maximum speed $v_{s}=8 Q_{2} /\left(\pi D^{2}\right)$ is reached at every section of the nozzle. If the kinetic energy supplied to the inner liquid is high enough, the resistance of the surface tension $\sigma$ is overcome, and the Couette-type flow in the needle tip gives rise downstream to a cylindrical jet of radius $R_{j}$. In both the gas-liquid and liquid-liquid configurations, the emitted jet eventually breaks up into droplets due to the growth of capillary waves. The breakup of gas-focused jets takes places out of the nozzle, while in the liquid-liquid configuration drops are produced in the nozzle's diverging part due to the destabilizing effect of the outer stream deceleration [20].

\subsection{Dimensionless parameters}

The focusing effect taking place in the needle tip depends on the device geometry, which includes both the nozzle shape and needle position. Here, we assume that the geometrical configuration is fixed, and discuss the relevance of the rest of the parameters involved in the problem.

Gaseous flow focusing can be characterized by the properties $\left\{\rho_{1}, \rho_{2}, \mu_{1}, \mu_{2}, \sigma\right\}$ of the fluids, and the control parameters $\left\{Q_{1}, \Delta p\right\}$. It must be noted that the focusing phenomenon is localized in a very small region as compared with the nozzle's characteristic size $D$, and thus this geometrical parameter does not enter our analysis. Alternatively, one considers the radius $R_{j}$ of the emitted jet, which is of the same order of magnitude as that of the needle tip, as the characteristic length of the problem. Five dimensionless numbers can be formed from this set of eight dimensional variables: the density $\rho_{2} / \rho_{1}$ and viscosity $\mu_{2} / \mu_{1}$ ratios, the Reynolds $\operatorname{Re}=\rho_{1} v_{j} R_{j} / \mu_{1}$ and Weber We $=\rho_{1} v_{j}^{2} R_{j} / \sigma$ numbers $\left(v_{j}=Q_{1} /\left(\pi R_{j}^{2}\right)\right.$ is the jet's average velocity), and the dimensionless group $C_{p}=$ $\mu_{1} /\left[\left(\rho_{1} \Delta p\right)^{1 / 2} R_{j}\right]$ associated with the pressure drop $\Delta p$. The density ratio takes small and nearly constant values for gasfocused liquids, and thus its influence can be neglected in the first-order approximation. It has been shown in previous works that the viscosity ratio does not affect significantly the stability of focused liquid tips. For instance, the tip curvature of a suspended drop at the bifurcation becomes independent of the outer-to-inner viscosity ratio if that ratio becomes smaller than about 0.1 (see, e.g., figure 8 in [21]). However, the shear viscous stress exerted by the gas stream is an essential element in dragging the film formed in the present technique, and thus the viscosity ratio $\mu_{2} / \mu_{1}$ should be retained in our analysis. The parameter $C_{p}$ takes very small values for sufficiently large applied pressure drops $\Delta p$, and thus it is assumed not to play a significant role in the stability analysis of most flow focusing applications [15]. This assumption will be validated experimentally in section 4.1. In sum, the stability analysis of the gas-focused jets can be analyzed in terms of the relevant dimensionless numbers $\mu_{2} / \mu_{1}, \operatorname{Re}$, and We.

The liquid-liquid flow focusing can be initially characterized by the properties $\left\{\rho_{1}, \rho_{2}, \mu_{1}, \mu_{2}, \sigma\right\}$ of the liquids, the control parameters $\left\{Q_{1}, Q_{2}\right\}$, and the local characteristic length $R_{j}$. Nevertheless, this set of parameters can be considerably simplified for the cases analyzed in the present work. In most of our experiments, the Reynolds numbers characterizing both the inner and outer streams are well below unity. In this case (and as pointed out by Gañán-Calvo [22]), the densities can be ruled out of the analysis, the jet stability becomes independent of its radius $R_{j}$, and the only relevant kinematic parameter is the interface velocity $v_{s}$. These assertions will be tested experimentally in section 4.2. Therefore, the stability analysis of the system can be performed in terms of the dimensional variables $\left\{\mu_{1}, \mu_{2}\right.$, $\left.\sigma, v_{s}\right\}$. Two dimensionless groups can be formed from these variables: the viscosity ratio $\mu_{2} / \mu_{1}$ and the capillary number $\mathrm{Ca}=\left(\mu_{1} \mu_{2}\right)^{1 / 2} v_{s} / \sigma[22]$.

\subsection{Stability analysis}

A rigorous stability analysis of any fluid configuration requires calculating its global modes [23], i.e., the temporal evolution of perturbations growing on the entire basic flow. In most practical applications, the calculation of a realistic basic flow constitutes a difficult problem in itself, because it involves the numerical integration of the three-dimensional nonlinear Navier-Sokes equations with complex boundary conditions. For this reason, one frequently simplifies the analysis by splitting the fluid domain into several regions. The stability of those regions that can be represented by simple basic flows are studied from linear stability analysis, while scaling laws are sought for the more complex parts of the fluid domain. The fluid configuration is assumed to be stable if and only if all its subdomains are stable. In flow focusing, one can clearly distinguish the liquid source from the emitted jet. Scaling analyses allow one to estimate the stability boundaries of the liquid source in some limiting cases [15], while the jet's behavior is described in terms of a spatial-temporal analysis of the dispersion relationship obtained for the appropriate basic flow $[10,24,25]$. This analysis entails the calculation of the critical parameters for which a transition from convective to absolute instability takes place in the jet [23]. In the convective instability regime, the perturbations are convected downstream for all wave numbers, while some of them travel upstream while growing in an absolutely unstable jet. The relationship between these two scenarios (convective and absolute instability) and the jetting and dripping regimes has been well established [23]. The calculation of the parameter points for which a basic flow undergoes a convective/absolute instability transition can be made tractable analytically at the expense of geometry simplifications.

As mentioned above, a transition from a threedimensional Couette-type flow to an axisymmetric capillary jet takes place in the tip of the needle used in the present technique. 


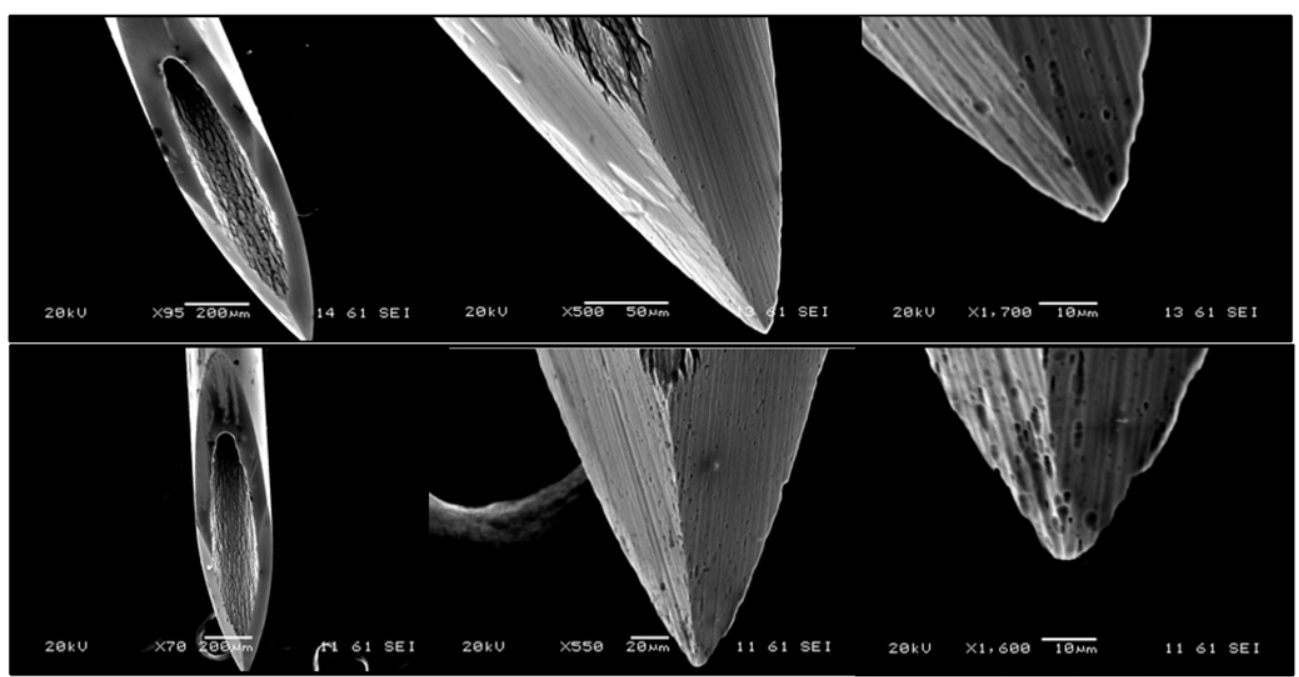

Figure 3. SEM images of two needles. The upper (lower) images correspond to a needle not chemically attacked (chemically attacked) with nitric acid. The acid deepens the existing micro-wells and pores, and creates new ones. The exposed surface area increases significantly.

Both shear and normal stresses exerted by the focusing stream collaborate in expelling the jet. While the viscous shear stress accelerates the inner stream over the needle's surface, the viscous/pressure normal stress stretches the interface overcoming the surface tension force. The magnitude of the shear and normal stresses increases as the velocity and acceleration of the outer stream increase, respectively. Therefore, the intensity of the focusing effect depends significantly on both the nozzle shape and the position of the needle tip in the nozzle. Because of the complexity of this phenomenon, one discards any rigorous theoretical analysis of its stability, and focuses on the jet's behavior exclusively. The stability requisites calculated this way must be regarded as necessary but not sufficient conditions for the system stability.

In gas-liquid systems, one usually neglects the effects of the coflowing gas stream on the jet's stability, and assumes that viscous diffusion of momentum flattens the jet's velocity profile. Then Leib and Goldstein's theory [26] provides good predictions for the convective/absolute instability transition taking place in the jet. It corresponds to a single curve (with no fitting variables) in the We-Re parameter plane. In the liquid-liquid configuration, the Reynolds number takes values well below unity for both the inner and outer streams. In this case, the jet's stability limit does not depend on the basic flow assumed for those streams. Gañán-Calvo [22] showed that the jet's stability can be described in terms of the capillary number. There is a critical value of that parameter above which the jet is convectively unstable. That value depends exclusively on the viscosity ratio. In section 4 , we will show that stable gas and liquid flow focusing realizations lie on the parameter regions delimited by the corresponding boundaries mentioned above.

\section{Experimental method}

The experimental apparatus is sketched in figure 5. We used an hypodermic needle (Becton Dickinson Microlance 3 30G 1/2) with an inner (outer) diameter of about 160 (300) $\mu \mathrm{m}$, and with an outer hydraulic radius $r_{t}$ of a few microns in the tip.
The needle tip was chemically attacked with nitric acid to enhance the liquid's capability of remaining attached to it. We performed a very mild etching, just to modify some aspects of the needle surface. The needle tip was dipped into nitric acid for a few tens of seconds, which produced two main effects (see figure 3). It enhanced the macroscopic surface roughness by deepening existing micro-wells and pores, and creating new ones, probably attacking the regions between metal grains. In this way, the exposed surface area increased significantly, which enhanced the hydrophilic nature of the metallic surface. In addition, the sharp edges of the needle tip were attacked to the point of leaving micro-spikes, some of them extremely thin. It must be noted that there is a marked variability among the tips of needles of the same brand and type, even among those taken from contiguous blister series. This variability is not leveled after nitric acid treatment (see figure 4).

The needle was located inside a converging-diverging nozzle (A) with a neck about $200 \mu \mathrm{m}$ in diameter by using high-precision orientation-translation systems (B). The nozzle was formed at one of the ends of a borosilicate capillary (BRAND), and its shape was characterized with the procedure described in [27]. The focused liquid was injected through the needle at a constant flow rate $Q_{1}$ by a syringe pump (HARVARD ApPARATUS PHD 4400) connected to a stepping motor. To produce the focusing effect with a gas stream, a constant pressure drop $\Delta p$ was applied to the surrounding air with a suction pump connected at the nozzle outlet. In the liquidliquid configuration, both the needle and the nozzle were immersed in a glycerine bath. The glycerine was suctioned across the nozzle at a constant flow rate $Q_{2}$ with another syringe pump.

Digital images of the fluid configuration were acquired using an ultra-high-speed CMOS camera (РHOTRON, FASTCAM SA5) (C) which allowed us to acquire images with an exposure time from $50 \mu$ s down to $340 \mathrm{~ns}$. The camera was equipped with a set of optical lenses (D). The optical lenses were selected depending on the size of the imaged object, with a magnification ranging from 0.076 to $0.52 \mu \mathrm{m} /$ pixel. This 


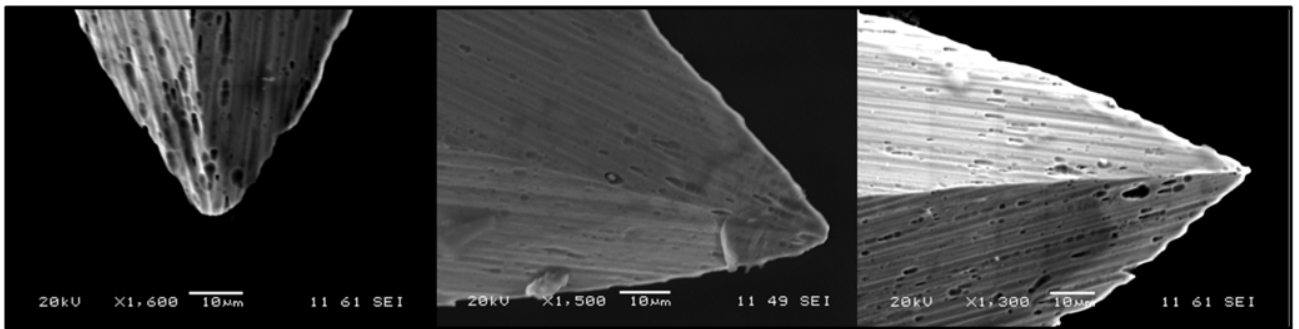

Figure 4. SEM images of three needles chemically attacked with nitric acid. There is a considerable variability among the needle tips.

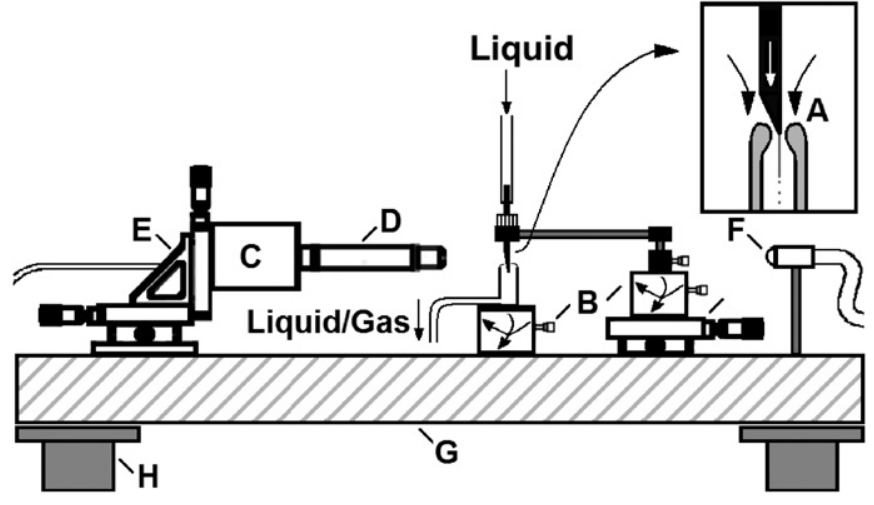

Figure 5. Experimental apparatus consisting of the following elements: needle located inside a converging-diverging nozzle (A), high-precision orientation-translation systems (B), digital camera (C), optical lenses (D), triaxial translation stage (E), optical fiber (F), optical table $(\mathrm{G})$, and pneumatic anti-vibration isolation system $(\mathrm{H})$.

parameter was calculated for each optical system by measuring the diameter of a calibration rod located in the field of view. In this way, the optical distortion caused by the glass nozzle was taken into account. The camera could be displaced both horizontally and vertically using a triaxial translation stage (E) to focus the jet. The fluid configuration was illuminated from the back side by cool white light provided by an optical fiber (F) connected to a light source. We also acquired images of the needle by using an auxiliary CCD camera (not shown in figure 5) with an optical axis perpendicular to that of the CMOS camera. The use of the two cameras allowed us to check that the needle was correctly positioned. All these elements were mounted on an optical table $(G)$ with a pneumatic antivibration isolation system $(\mathrm{H})$ to damp the vibrations coming from the building. By way of illustration, figure 6 shows two images acquired in the course of the experiments. The left-hand image corresponds to a gas-focused jet of radius $R_{j} \simeq 1.4 \mu \mathrm{m}$, while the photograph on the right-hand side shows both the tapering cone hanging from the needle tip and the emitted jet in a liquid-liquid configuration.

Table 1 shows the values of the properties of the working liquids, and the symbols used in the rest of the paper. The surface tension was measured with the theoretical image fitting analysis (TIFA) method [28], while the rest of the liquid properties were taken from the manufacture's specifications. Table 2 displays the surface tension value of the interface between the silicone oils and glycerine also measured with the TIFA method [28].
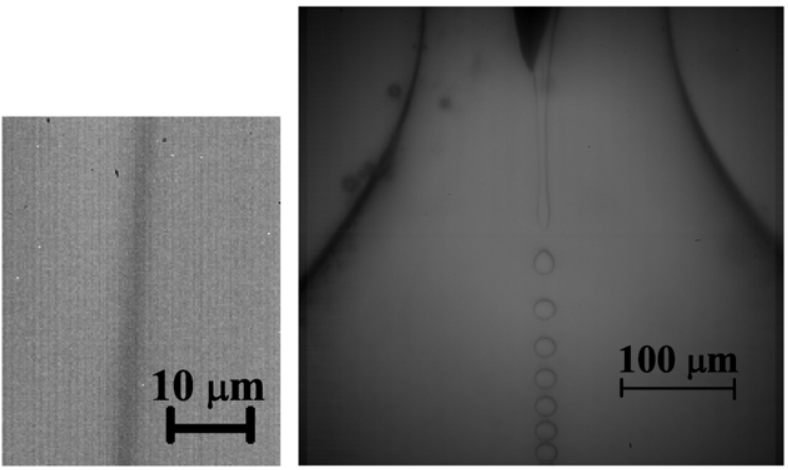

Figure 6. (Left) Gas-focused jet of $5 \mathrm{cSt}$ silicone oil produced with $Q_{1}=90 \mu \mathrm{lh}^{-1}$ and $\Delta p=250 \mathrm{mbar}$. (Right) Tapering cone of $5 \mathrm{cSt}$ silicone oil and the resulting jet flowing at $Q_{1}=0.5 \mu \mathrm{h} \mathrm{h}^{-1}$ inside a glycerol stream with a flow rate $Q_{2}=13 \mathrm{ml} \mathrm{h}^{-1}$.

Table 1. Properties of the working liquids at $20^{\circ} \mathrm{C}$, and the symbols used in the rest of the paper.

\begin{tabular}{lclll}
\hline Liquid & $\rho\left(\mathrm{kg} \mathrm{m}^{-3}\right)$ & $\sigma\left(\mathrm{mJ} \mathrm{m}^{-2}\right)$ & $\mu(\mathrm{Pa} \mathrm{s})$ & Symbol \\
\hline 0.65 cSt silicone oil & 740 & $17 \pm 1$ & 0.000481 & \\
1 cSt silicone oil & 818 & $17 \pm 1$ & 0.00082 & $\bullet$ \\
5 cSt silicone oil & 917 & $19 \pm 1$ & 0.0046 & $\mathbf{\square}$ \\
35 cSt silicone oil & 957 & $20 \pm 1$ & 0.033 & $\mathbf{}$ \\
100 cSt silicone oil & 960 & $21 \pm 1$ & 0.096 & $\vdots$ \\
350 cSt silicone oil & 970 & $21 \pm 1$ & 0.34 & $\star$ \\
500 cSt silicone oil & 970 & $20 \pm 1$ & 0.48 & $\mathbf{\nabla}$ \\
Water & 998 & $72 \pm 1$ & 0.001 & $\square$ \\
Glycerine & 1257 & $63 \pm 1$ & 1.5 & \\
\hline
\end{tabular}

\section{Results}

\subsection{Gas-focused jets}

In this section, we present and discuss the results obtained for gas-focused jets with two needles, N1 and N2, of hydraulic radius at the tip $r_{t} \simeq 0.6$ and $2.0 \mu \mathrm{m}$, respectively. The needle tip was located in the nozzle neck, a constant pressure drop was applied to the air stream, and the liquid flow rate was progressively decreased until the transition from the jetting to the dripping regime took place. The minimum flow rates measured for varied applied pressure drops are shown in figure 7 . The figure also shows the results obtained with the optimum configuration of the standard flow focusing technique [15]. The present and the standard flow focusing configurations exhibit similar behaviors. For relatively small values of the pressure drop, the minimum flow rate decreases as the pressure 
Table 2. Surface tension of the interface liquid-glycerol for the liquids used in the experiments.

\begin{tabular}{llllllll}
\hline Liquid & $0.65 \mathrm{cSt} \mathrm{SO}$ & $1 \mathrm{cSt} \mathrm{SO}$ & $5 \mathrm{cSt} \mathrm{SO}$ & $35 \mathrm{cSt} \mathrm{SO}$ & $100 \mathrm{cSt} \mathrm{SO}$ & $350 \mathrm{cSt} \mathrm{SO}$ & $500 \mathrm{cSt} \mathrm{SO}$ \\
\hline$\sigma\left(\mathrm{mJ} \mathrm{m}^{-2}\right)$ & $27 \pm 1$ & $27 \pm 1$ & $27 \pm 1$ & $27 \pm 1$ & $26 \pm 1$ & $27 \pm 1$ & $27 \pm 1$ \\
\hline
\end{tabular}

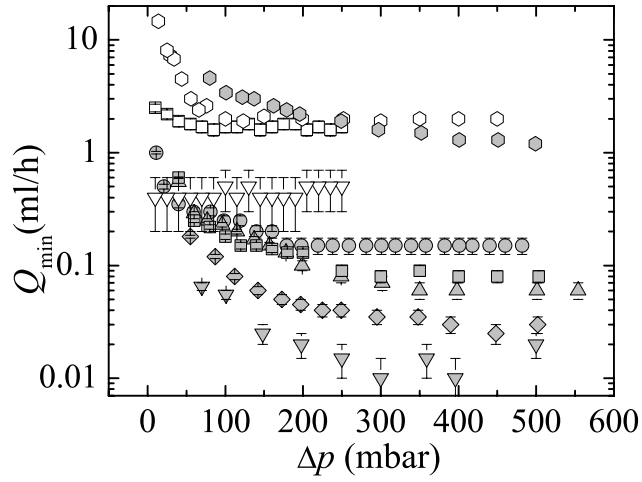

Figure 7. The minimum flow rate $Q_{\min }$ versus the applied pressure drop $\Delta p$ for the needle N2 (gray symbols). The white symbols are the minimum flow rates obtained in [15] with the optimum plate-orifice configuration.

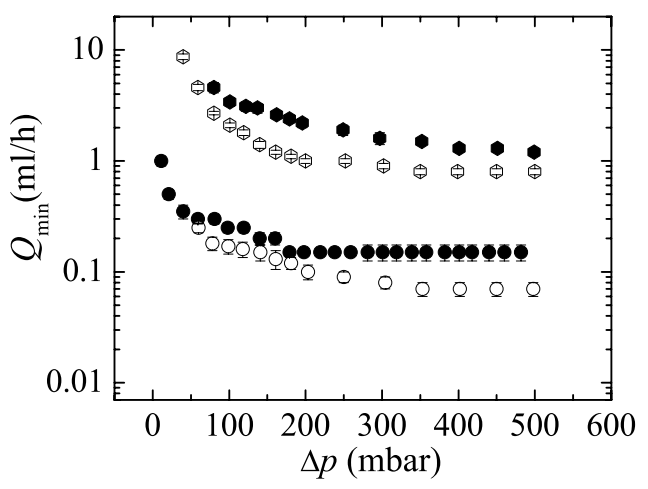

Figure 8. The minimum flow rate $Q_{\text {min }}$ versus the applied pressure drop $\Delta p$ for the needles N1 (white symbols) and N2 (black symbols).

drop increases. For sufficiently large values of $\Delta p$, the flow rate threshold becomes independent of that parameter. The fluidic structure in the needle tip proves to be much more stable than the tapering meniscus in the standard configuration for the whole range of viscosities analyzed. In fact, the minimum flow rates reached with the present technique are two orders of magnitude smaller than their counterparts in the classical configuration. Water constitutes an exception owing to its high surface tension value. In this case, both techniques lead to similar results. The dependence of the minimum flow rate on the needle tip radius $r_{t}$ is analyzed in figure 8 . As can be observed, the minimum flow rate decreases with $r_{t}$, which shows the influence of the Couette flow to jet transition on the system stability.

The remarkable stability of the liquid source in the proposed technique allows one to produce extremely small jets. Table 3 displays the values of the radius $R_{j}$ of jets emitted at the minimum flow rate. The results for $100 \mathrm{cSt}$ and $500 \mathrm{cSt}$ silicone oils are not presented because we could not get reliable measurements due to the jet's smallness. The
Table 3. Jet radius measured in microns for jets focused at the minimum flow rate with the needles $\mathrm{N} 1$ and $\mathrm{N} 2$ for $\Delta p=75$ and $250 \mathrm{mbar}$. The diameters for $100 \mathrm{cSt}$ silicone oil and $500 \mathrm{cSt}$ silicone oil were too small to get reliable values.

\begin{tabular}{llllll}
\hline Needle & $\Delta p$ (mbar) & Water & $1 \mathrm{cSt} \mathrm{SO}$ & $5 \mathrm{cSt} \mathrm{SO}$ & $35 \mathrm{cSt} \mathrm{SO}$ \\
\hline N1 & 75 & 7.8 & 1.6 & 1.6 & 1.5 \\
N1 & 250 & 3.3 & 0.9 & 0.8 & 0.8 \\
N2 & 250 & 4.0 & 2.3 & 1.4 & 0.9 \\
\hline
\end{tabular}

jets are two orders of magnitude thinner than any orifice of the configuration used to produce them. The radii reached the submicrometer range, a remarkable achievement for jets produced in a gaseous ambient by purely hydrodynamic means. The radii for $\Delta p=250$ mbar were significantly smaller than those measured for $\Delta p=75$ mbar because lower minimum flow rates were reached in the former case (figure 7). The jet's radius reached values even smaller than $r_{t}$ with the needle N2, which implies that the free surface was stretched downstream by the gas stream in that case. The jet's radius decreased with the needle tip size $r_{t}$. The needle N1 was so sharp that its size constituted a barrier for the jet's radius.

One can provide the above experimental results with a qualitative theoretical basis. To this end, one estimates the thickness $w$ of the Couette stream formed next to the needle tip. We shall assume that the free surface velocity $v_{s}$ is much smaller than the velocity $v_{2} \sim\left(\Delta p / \rho_{2}\right)^{1 / 2}$ of the gas stream. In this case, the integral form of the momentum equation for the accelerated gas boundary layer leads to $\rho_{2} v_{2}^{2} H^{-1} \sim \mu_{2} v_{2} / \delta_{2}^{2}$, where $H$ is a streamwise characteristic length $(H \sim 100 \mu \mathrm{m}$ in our experiments), and $\delta_{2}$ is the characteristic boundary layer thickness. The balance of shear stresses on the two sides of the meniscus free surface yields $\mu_{1} v_{s} / w \sim \mu_{2} v_{2} / \delta_{2}$. From the above two equations, one concludes that the free surface velocity scales as $v_{s} \sim w \mu_{1}^{-1}\left(\rho_{2} \mu_{2} v_{2}^{3} H^{-1}\right)^{1 / 2}$. If one takes into account that $Q \sim v_{s} w r_{t}$, then

$$
w \sim\left(Q \mu_{1} / r_{t}\right)^{1 / 2}\left(\rho_{2} \mu_{2} v_{2}^{3} H^{-1}\right)^{-1 / 4} .
$$

In addition, $R_{j} \sim\left(r_{t} w\right)^{1 / 2}$ because the jet's velocity is of the order of $v_{s}$. It can be seen that $r_{t} \sim w \sim R_{j} \sim 1 \mu \mathrm{m}$ for the minimum flow rates measured in the experiments, which agrees with the results presented in table 3 .

Figure 9 shows the critical Weber numbers for those liquids whose radius could be measured. The solid line is the Leib and Goldstein prediction [26] for the convective/absolute instability transition [23]. The critical Weber number significantly exceeded that prediction, which indicates that the loss of stability must be attributed to the liquid source. This occurred in all the cases except those of $1 \mathrm{cSt}$ silicone oil for $\mathrm{N} 2$ and $\Delta p=250 \mathrm{mbar}$, and water for $\mathrm{N} 1$ and $\Delta p=75$ mbar. In these cases, the jet stability limit coincided with or dominated that associated with the liquid source. The critical Weber number corresponding to $1 \mathrm{cSt}$ silicone oil 


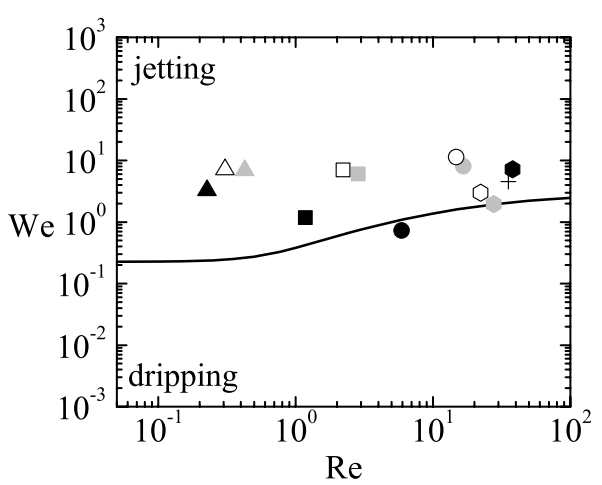

Figure 9. Critical values of the Weber number with the needle N1 for $\Delta p=75$ mbar (gray symbols) and $250 \mathrm{mbar}$ (white symbols), and with the needle $\mathrm{N} 2$ for $\Delta p=250 \mathrm{mbar}$ (black symbols). The cross corresponds to water focused with the standard flow focusing configuration [25]. The solid line is the Leib and Goldstein prediction [26] for the convective/absolute instability transition. The diameters for $100 \mathrm{cSt}$ silicone oil and $500 \mathrm{cSt}$ silicone oil were too small to be measured, and thus the Reynolds and Weber numbers could not be estimated.

is slightly below the jet's stability curve. The fact that the experimental point slightly surpasses the jet stability limit can be attributed to either the limitation of the theoretical model or experimental uncertainty. On one side, the uniform basic flow underestimates the free surface velocity, and thus Leib and Goldstein's prediction overestimates the critical Weber number. On the other side, an error of just $10 \%$ in measuring the jet radius explains the discrepancy of about $30 \%$ between the theoretical and experimental values of the critical Weber number. The critical Weber and Reynolds numbers increased as the needle tip size decreased because the jet's radius significantly decreased with the tip size (table 3 ). Water constitutes again an exception because its minimum flow rate significantly decreased (figure 8 ) with the tip size.

A quantitative analysis of the liquid source stability is really complex. If the needle surface had irregularities with sizes of the order of the liquid film thickness $(1 \mu \mathrm{m})$, then they would stall the flow and prevent the jetting regime from being reached. Assume that such irregularities do not exist. Then, instability would be primarily caused by the surface tension force at the needle tip. As the flow rate of the injected liquid decreases, the film thickness decreases, and the capillary force increases. For a sufficiently small flow rate, surface tension overcomes inertia, and the liquid ejection is interrupted. Here, the liquid's capability of remaining attached to the needle surface plays a crucial role as the stream flows toward the tip. Those liquids which detach earlier will do so with lower Weber numbers, and thus will drip at larger flow rates. It must be noted that the Weber number at the jet's emission point can be significantly lower than that measured when the jet is completely formed (figure 9). The liquid's capability of remaining attached to the needle surface depends not only on its density and viscosity but also on the wettability process, which greatly hinders any quantitative analysis.

Figure 10 illustrates the improvement of the present technique with respect to previous flow focusing configurations. The figure shows the stability map in the parameter plane defined by the dimensionless numbers $Q / Q_{D}$ and

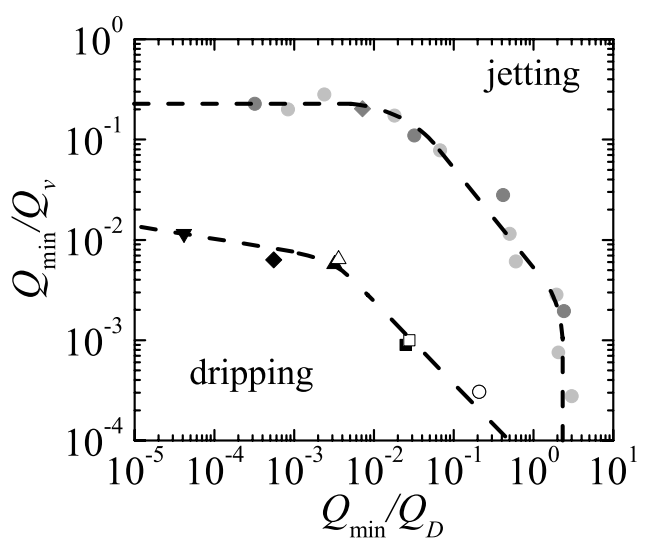

Figure 10. $Q_{\min } / Q_{D}$ versus $Q_{\min } / Q_{v}$ obtained with the needle N1 (white symbols) and N2 (black symbols) for $\Delta p=250 \mathrm{mbar}$ and the liquids listed in table 1 . In some cases, black and white symbols overlap. The light-gray and gray symbols are the limiting flow rates obtained in [15] and [29] with the standard plate-orifice configuration and the converging-diverging nozzle, respectively. The dashed lines are to guide the eye.

$Q / Q_{v}$ characterizing the tapering meniscus stability in the standard flow focusing configuration. Here, $Q_{D}=D \mu_{1} / \rho_{1}$ and $Q_{v}=\sigma D^{2} /\left(4 \mu_{1}\right)$ are the characteristic flow rates for that configuration in the inviscid and viscous limits, respectively [15]. These characteristic flow rates were obtained by considering the mechanisms responsible for the instability of the flow focusing meniscus, i.e., the energy dissipation in the recirculation cell for low-viscosity liquids, and the retraction force associated with the surface tension in the viscous case [15]. As can be observed in figure 10, the liquid injection system proposed in this work drastically compresses the dripping region. The fact that $Q_{\min } / Q_{D}$ and $Q_{\min } / Q_{v}$ take values much lower than unity in the proposed configuration indicates that the instability mechanism is completely different from those of the classical flow focusing configuration.

\subsection{Liquid-focused jets}

The liquid-liquid configuration is studied in this section. The results were obtained with the needle N3 of outer hydraulic radius $r_{t}=4.5 \mu \mathrm{m}$. In order to simplify the stability analysis of this configuration, the needle tip was located in the nozzle neck, which eliminates the effect of the outer stream acceleration. Then the flow rate $Q_{1}$ of the inner stream was fixed, and the flow rate $Q_{2}$ of the outer stream was gradually lowered until the transition from the jetting to the dripping regime took place. Figure 11 shows the critical value $Q_{2 \text {,min }}$ of the focusing flow rate $Q_{2}$ as a function of the focused flow rate $Q_{1}$. The stability threshold $Q_{2 \text {,min }}$ does not depend on $Q_{1}$ for sufficiently small values of this parameter.

In our experiments, the jet's Reynolds number takes small values, viscous diffusion flattens the jet's velocity profile, and thus the jet's average velocity $v_{j}$ coincides with that of the free surface $v_{s}$. Because the outer stream is very viscous, its velocity $v_{2, \max }=8 Q_{2} /\left(\pi D^{2}\right)$ at the nozzle axis equals $v_{s}$, and thus $v_{j}$. Therefore, the jet's radius $R_{j}$ can be calculated as

$$
R_{j}=\frac{\left(Q_{1} / Q_{2}\right)^{1 / 2} D}{\sqrt{8}},
$$




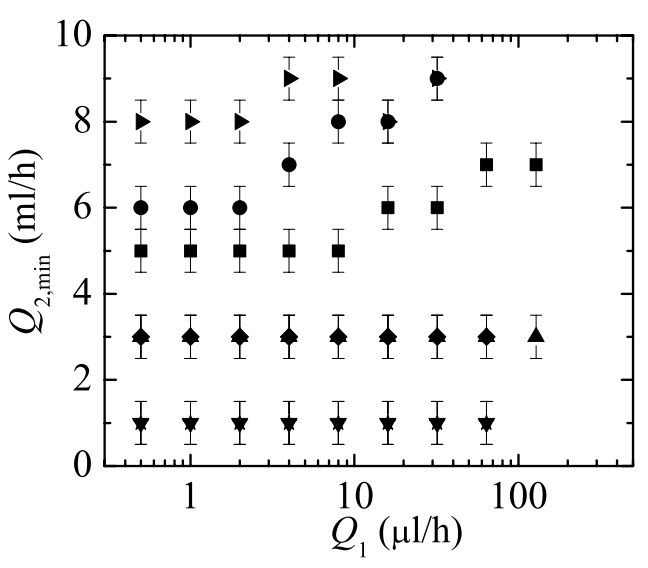

Figure 11. Minimum values $Q_{2 \text {,min }}$ of the focusing flow rate versus the focused flow rate $Q_{1}$.

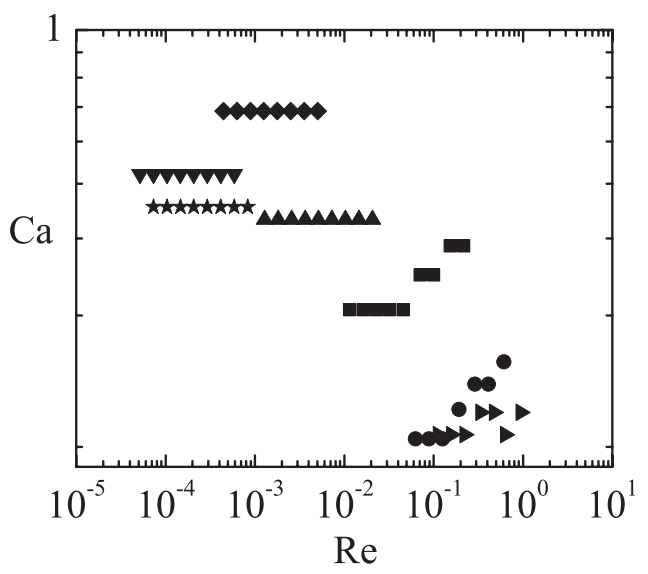

Figure 12. Critical values of the capillary number $\mathrm{Ca}$ versus the Reynolds number Re.

while the Reynolds and capillary numbers can be written as

$$
\operatorname{Re}=\frac{\rho_{1}\left(Q_{1} Q_{2}\right)^{1 / 2}}{\pi \mu_{1} D} \text { and } \mathrm{Ca}=\frac{8\left(\mu_{1} \mu_{2}\right)^{1 / 2} Q_{2}}{\pi D^{2} \sigma} .
$$

Figure 12 shows the critical capillary number as a function of the Reynolds number. The instability limit becomes independent of the Reynolds number for $\operatorname{Re} \lesssim 0.1$, which indicates the beginning of the Stokes regime. In this regime, $R_{j}$ depends exclusively on $Q_{1}$ for a given value of $Q_{2}$. Therefore, if $Q_{2 \text {,min }}$ remains constant while varying $Q_{1}$ (figure 11), then one concludes that $Q_{2 \text {,min }}$ does not depend on $R_{j}$ either. In this case, infinitely thin jets could be produced provided that $Q_{2}$ exceeded its threshold value $Q_{2 \text {,min }}$. This possibility has been identified by Gañán-Calvo [22] from the analysis of the convective/absolute instability transition with dominant viscosity.

The critical values of the capillary number in the Stokes regime are plotted in figure 13. The figure also shows the theoretical prediction for the convective/absolute instability transition in the emitted jet [22]. As can be observed, the critical Capillary number of most liquids was notably greater than the corresponding theoretical value. In all those cases, the loss of stability must be attributed to the liquid source, and not to the jet itself. The stability limits of $0.65 \mathrm{cSt}$ and $1 \mathrm{cSt}$ silicone oils were accurately predicted by the convective/absolute

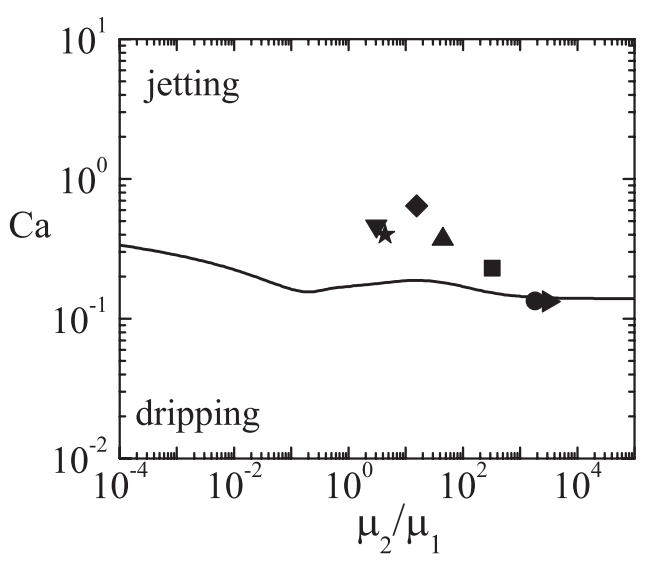

Figure 13. Critical values of the capillary number Ca versus the viscosity ratio $\mu_{2} / \mu_{1}$.

instability transition occurring in the emitted jet [22]. In these cases, the jet stability limit coincided with or dominated that associated with the liquid source. Interestingly, the critical capillary number scales as $\left(\mu_{2} / \mu_{1}\right)^{-1 / 3}$ for large values of $\mu_{2} / \mu_{1}$, and then reaches a maximum for $\mu_{2} / \mu_{1} \sim 10$.

The size of droplets produced with the present technique can be precisely controlled by shifting the needle tip along the nozzle without varying the flow rates. Figure 14 shows the radius $R_{d}$ of the droplets produced by focusing $5 \mathrm{cSt}$ silicone oil with glycerol. The radius $R_{d}$ is plotted as a function of the distance $z$ between the needle tip and the nozzle neck. In the nozzle converging part $(z<0), R_{d}$ decreases as the distance from the neck increases. The opposite occurs in the nozzle diverging region. In both cases, one can appreciate the influence of the extensional viscous stresses exerted by the outer stream. In the converging part (figure 14 (left)), the outer stream speeds up, and thus viscous stresses stretch the meniscus hanging from the needle tip. This focusing effect reduces very considerably the emitted jet size, which can go down to values hardly observable with standard microscopy. The 'invisible' jet moves downstream until it reaches the diverging region. There, the viscous stresses exerted by the slowing outer stream oppose the jet's motion, which destabilizes the jet and makes it break up into droplets [20]. Due to the extensional viscous stresses and the disparity between the inner and outer viscosities, the droplet radius is much larger than that of the jet [11], which makes droplets visible. If the needle tip is located in the nozzle diverging region, viscous stresses collaborate with surface tension in destabilizing the meniscus. If the inner liquid kinetic energy is high enough to overcome those destabilizing factors, the meniscus still forms and steadily ejects a short liquid ligament. This liquid ligament immediately breaks up into droplets of tens of microns in diameter.

In figure 15 , we zoomed in on the $5 \mathrm{cSt}$ silicone oil meniscus formed in an experiment with $Q_{1}=0.05 \mu \mathrm{l}$, the lowest flow rate reachable with our experimental apparatus. This flow rate is two orders of magnitude less than those obtained with the double flow focusing configuration [19], and five orders of magnitude less than those reported when using the standard liquid-liquid flow focusing technique [10]. 

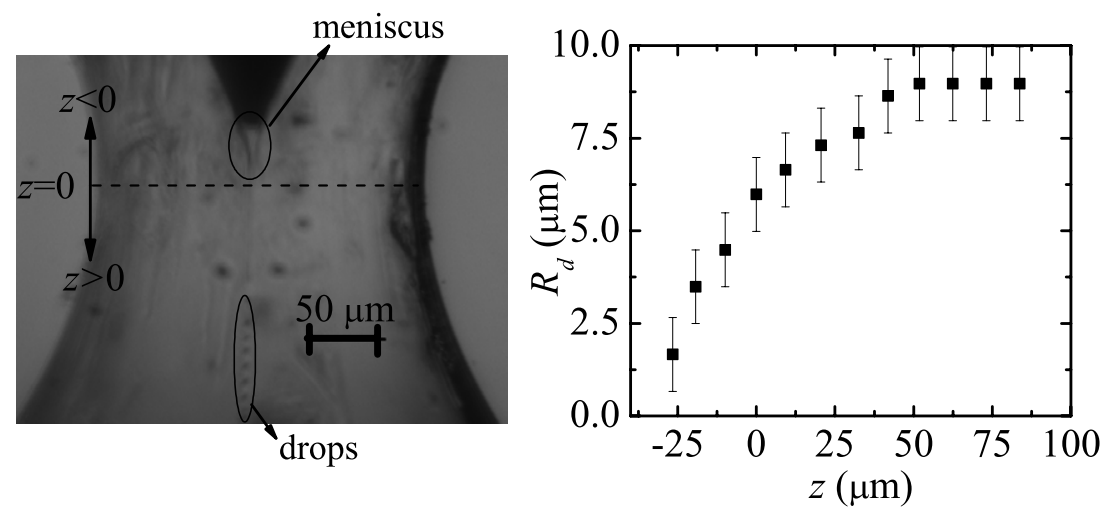

Figure 14. Radius $R_{d}$ of the produced droplets by focusing $5 \mathrm{cSt}$ silicone oil with glycerol with $Q_{1}=0.5 \mu 1 \mathrm{~h}^{-1}$ and $Q_{2}=12 \mathrm{mlh}^{-1}$.

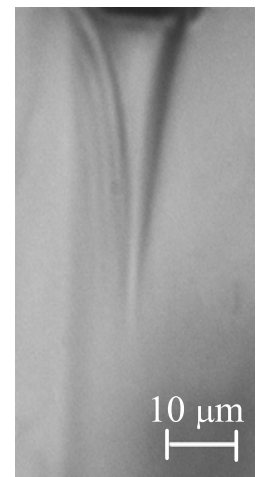

Figure 15. $5 \mathrm{cSt}$ silicone oil meniscus formed in an experiment with $Q_{1}=0.05 \mu 1 \mathrm{~h}^{-1}$ and $Q_{2}=10 \mathrm{ml} \mathrm{h}^{-1}$.

The meniscus was in the shape of a cone whose tip can not be appreciated despite the large magnification used in the experiment. An invisible submicrometer jet was emitted from the cone's tip. Its existence is confirmed by the appearance downstream of drops of about $1 \mu \mathrm{m}$ in diameter.

\section{Conclusions}

We have presented a novel technique for producing very thin jets in both the gas-liquid and the liquid-liquid configurations. A film is formed on the outer surface of a hypodermic needle by injecting liquid at a constant flow rate. A coflowing fluid stream drags the film to the very tip of the needle, which remains attached to the needle outer surface until it reaches the tip. This film-type flow is mainly responsible for the reduction of the jet's size, and provides it with the momentum necessary to overcome the surface tension. There can also be a slight jet elongation in the needle tip depending on the specific fluidliquid configuration considered, but this elongation produces a reduction of the jet's diameter much less than that occurring over the film-type flow. The proposed method benefits from the remarkable stability of the fluidic structure from which the jets originate. This fluidic structure turns out to be very stable as the injected flow rate decreases. In fact, one can steadily form jets at rates of flow two orders of magnitude smaller than the limiting values in flow focusing $[15,19]$. In this way, liquid jets are produced with radii down to the submicrometer scale by purely hydrodynamic means. The flow in this technique is essentially different from that taking place in the standard flow focusing configuration. In this latter case, a meniscus held by surface tension is stretched by the outer current until it emits a very thin jet from its apex. The liquid current elongates without touching any solid surface. This process is more energetically efficient than that occurring in the needle, but prone to instability as the injected flow rate decreases.

The present experimental study has mainly focused on the stability of the proposed technique. The main conclusions for the gas-liquid configuration are:

- The dependence of the minimum flow rate upon the pressure drop applied to the gas stream was similar to that of the flow focusing technique: the minimum flow rate decreased as the pressure drop increased for relatively small values of $\Delta p$, and it became independent of the pressure drop for sufficiently large values of this last parameter.

- The minimum flow rate decreased with the size of the needle tip.

- The critical Weber and Reynolds numbers increased as the needle tip size decreased because the jet's radius significantly decreased with the tip size.

The experiments conducted with the liquid-liquid configuration allowed us to come to the following conclusions.

- The minimum value of the outer flow rate did not depend on the inner one for sufficiently small values of this last parameter. Therefore, infinitely thin jets could be produced in the limit of vanishing inner flow rates [22] as long as the outer flow rate exceeded its threshold value.

- The Stokes regime was reached in our experiments, where the instability limit becomes independent of the Reynolds number.

- Invisible jets were emitted at the lowest flow rate attainable with our experimental apparatus. Their existence had to be confirmed by the appearance downstream of drops of about $1 \mu \mathrm{m}$ in diameter.

As explained in section 2.3 , the origin of the instability in the limit of small flow rates was identified by projecting the experimental data onto the parameter plane characterizing the jet's behavior. In this way, one can distinguish whether the instability originates at the liquid source or at the emitted jet. In most of the cases, the loss of stability must be attributed to the 
liquid source because the critical value of the Weber/Capillary number significantly exceeded that corresponding to the convective-absolute instability transition in the jet.

The liquid source in the proposed technique is much more stable than in the classical flow focusing configuration as the flow rate decreases. This stability improvement entails, however, a certain limitation in controlling the magnitude of the focusing effect, and, therefore, the emitted jet's size. In order to better control the magnitude of the focusing effect, the needle is put into a converging-diverging nozzle [29]. Because the axial velocity and acceleration of the outer stream depend on the nozzle section, the resulting jet's radius depends significantly on the location of the needle tip inside the nozzle. For instance, our experiments for liquid-liquid systems showed that submicrometer jets can be produced when the needle tip is located in front of the neck, while liquid ligaments of tens of microns can be obtained if the tip is positioned behind the neck. Therefore, the size of the resulting droplets can be precisely determined by shifting the needle tip along the nozzle without varying the flow rates.

\section{Acknowledgments}

Partial support from the Ministry of Science and Education, Junta de Extremadura, and Junta de Andalucía (Spain) through grants nos DPI2010-21103, GR10047, and P08-TEP-04128, respectively, is gratefully acknowledged.

\section{References}

[1] Eggleton C D, Tsai T-M and Stebe K J 2001 Tip streaming from a drop in the presence of surfactants Phys. Rev. Lett. 87048302

[2] Fernández de la Mora J 2007 The fluid dynamics of Taylor cones Annu. Rev. Fluid Mech. 39 217-43

[3] Basaran O A 2002 Small-scale free surface flows with breakup: drop formation and emerging applications AIChE J. 48 1842-8

[4] Gañán-Calvo A M 1998 Generation of steady liquid microthreads and micron-sized monodisperse sprays in gas streams Phys. Rev. Lett. 80 285-8

[5] Cohen I, Li H, Hougland J L, Mrksich M and Nagel S R 2001 Using selective withdrawal to coat microparticles Science 292 265-7

[6] Utada A S, Lorenceau E, Link D R, Kaplan P D, Stone H A and Weitz D A 2005 Monodisperse double emulsions generated from a microcapillary device Science $308537-41$

[7] Takeuchi S, Garstecki P, Weibel D B and Whitesides G M 2005 An axisymmetric flow-focusing microfluidic device Adv. Mater. 17 1067-72

[8] Blanchette F and Zhang W W 2009 Force balance at the transition from selective withdrawal to viscous entrainment Phys. Rev. Lett. 102144501

[9] Rayleigh L 1879 On the instability of jets Proc. Lond. Math. Soc. $104-13$
[10] Gañán-Calvo A M and Riesco-Chueca P 2006 Jetting-dripping transition of a liquid jet in a lower viscosity co-flowing immiscible liquid: the minimum flow rate in flow focusing J. Fluid Mech. 553 75-84

[11] Tomotika S 1935 On the instability of a cylindrical thread of a viscous liquid surrounded by another viscous liquid Proc. R. Soc. Lond. 150 322-37

[12] Gañán-Calvo A M, Pérez-Saborid M, López-Herrera J M and Gordillo J M 2004 Steady high viscosity liquid micro-jet production and fiber spinning using co-flowing gas conformation Eur. Phys. J. B 39 131-7

[13] Eggers J and Villermaux E 2008 Physics of liquid jets Rep. Prog. Phys. 71036601

[14] Herrada M A, Gañán-Calvo A M, Ojeda-Monge A, Bluth B and Riesco-Chueca P 2008 Liquid flow focused by a gas: jetting, dripping, and recirculation Phys. Rev. E 78036323

[15] Montanero J M, Rebollo-Muñoz N, Herrada M A and Gañán-Calvo A M 2011 Global stability of the focusing effect of fluid jet flows Phys. Rev. E 82036309

[16] Acero A J, Montanero J M, Ferrera C, Herrada M A and Gañán-Calvo A M 2012 Enhancement of the stability of the flow focusing technique for low-viscosity liquids J. Micromech. Microeng. 22115039

[17] Whitehouse C M, Dreyer R N, Yamashita M and Fenn J B 1985 Electrospray interface for liquid chromatographs and mass spectrometers Anal. Chem. 57 675-9

[18] Fenn J B, Mann M, Meng C K, Wong S F and Whitehouse C M 1989 Electrospray ionization for mass spectrometry of large biomolecules Science 246 64-71

[19] Gañán-Calvo A M, González-Prieto R, Riesco-Chueca P, Herrada M A and Flores-Mosquera M 2007 Focusing capillary jets close to the continuum limit Nature Phys. $3737-42$

[20] Tomotika S 1936 Breaking up of a drop of viscous liquid immersed in another viscous fluid which is extending at a uniform rate Proc. R. Soc. Lond. 153 302-18

[21] Eggers J and du Pont S C 2009 Numerical analysis of tips in viscous flow Phys. Rev. E 79066311

[22] Gañán-Calvo A M 2008 Unconditional jetting Phys. Rev. E 78026304

[23] Huerre P and Monkewitz P A 1990 Local and global instabilities in spatially developing flows Annu. Rev. Fluid Mech. 22 473-537

[24] Si T, Li F, Yin X-Y and Yin X-Z 2009 Modes in flow focusing and instability of coaxial liquid-gas jets J. Fluid Mech. 629 1-23

[25] Vega E J, Montanero J M, Herrada M A and Gañán-Calvo A M 2010 Global and local instability of flow focusing: the influence of the geometry Phys. Fluids 22064105

[26] Leib S J and Goldstein M E 1986 Convective and absolute instability of a viscous liquid jet Phys. Fluids 29 952-4

[27] Montanero J M, Gañán-Calvo A M, Acero A J and Vega E J 2010 Micrometer glass nozzles for flow focusing J. Micromech. Microeng. 20075035

[28] Cabezas M G, Bateni A, Montanero J M and Neumann A W 2005 A new method of image processing in the analysis of axisymmetric drop shapes Colloids Surf. A 255 193-200

[29] Acero A J, Ferrera C, Montanero J M and Gañán-Calvo A M 2012 Focusing liquid microjets with nozzles J. Micromech. Microeng. 22065011 